\title{
Predictibilidad a corto plazo del número de casos de la influenza pandémica AH1N1 basada en modelos determinísticos
}

\author{
Mauricio Canals L.
}

\section{Short-term predictability of influenza AH1N1 cases based on deterministic models}

Influenza AH1N1 severely affected the population of Chile. It has high transmissibility, which may stress the health system capacity. An adequate prediction of number of cases and frequency of complications is needed. Chilean and OMS dialy reports of cases from April to Jun 2009 were analyzed in this study. We developed a simple methodology for short-term forecast of case number, based on deterministic models. Predicted and observed values were compared with regression analyses and Bland-Altman diagrams. We found that the intrinsic rate of increase of the number of cases in the world and in Chile, after an initial high instability, decreases to stable values, allowing a relatively accurate forecasting the next day and for a serial period. The estimators obtained are over-estimators, which allow an adequate safety factor for the estimations. The method is easy to implement in software for routine use and can be useful in monitoring this epidemic and others in the future.

Key words: Influenza, forecast, deterministic models.

Palabras clave: Influenza, predicción, modelos determinísticos.

\section{Introducción}

$\mathrm{L}$ a emergencia de pandemias de influenza, una enfermedad zoonótica, es un hecho común que de cuando en cuando azota a la humanidad. Así por ejemplo, la pandemia más grave descrita, la llamada influenza "española" (AH1N1) ocurrió entre 1918 y 1919 y durante su transcurso murieron aproximadamente 21 millones de personas ${ }^{1-3}$. Sin embargo, han ocurrido otras como el virus asiático en 1957-1958 (AH2N2), "la gripe de Hong-Kong" (AH3N2) en 1968-1969 y otra de tipo AH1N1 en 1977, que aunque han contagiado a muchas personas, han tenido una mortalidad menor ${ }^{2}$. El virus tiene una capacidad mutagénica importante y un número reproductivo $\left(R_{0}\right)$ cercano a 2 , moderado a bajo en comparación con otras enfermedades infecciosas como el sarampión y otras ${ }^{3-6}$. Sin embargo, este es suficiente para propagar la infección rápidamente y comprometer gran cantidad de población. La mantención del virus en el reservorio animal permite que estas epidemias resurjan cada cierto tiempo, alrededor de 9 a 39 años en el caso de la influenza 2 .

Así, el mundo enfrenta hoy una pandemia A H1N1 que en la actualidad ya lleva más de 60 mil casos notifi$\operatorname{cados}^{7}$. Sea cual sea la morbi-mortalidad que tenga, esta epidemia amenaza con colapsar los sistemas de salud por el aumento de casos. La respuesta del o los sistemas de salud, fuera de las medidas de tratamiento adecuado y oportuno de los casos individuales, debe incluir como uno de los factores principales la estimación del número de casos para apoyar la planificación de la respuesta y la contención de la epidemia, cortando o retardando la cadena de transmisión. La predicción de los posibles casos es, no obstante, dificultosa, ya que en general al inicio de la epidemia no se conoce el número de susceptibles ni el número reproductivo ${ }^{8}$, un parámetro fundamental para conocer la transmisibilidad de una epidemia y el número de casos subsecuentes, que para enfermedades infecciosas emergentes nuevas sólo puede ser estimado indirectamente a partir de la tasa de crecimiento epidémica observada $(R t)$. A pesar de ello se cuenta desde inicios del siglo 20 con un acabado conocimiento de la dinámica epidémica y de las ecuaciones que la determinan 9 . Se conocen la relación entre la incidencia y el número de casos acumulados y la tasa intrínseca de producción de casos y su relación con el número reproductivo básico (ie número de casos nuevos a partir de un caso índice en un período serial) y a partir de estas relaciones es posible establecer una predictibilidad a corto plazo que permite adoptar medidas de salud pública. En este trabajo, basados en modelos determinísticos básicos, estudiamos la predictibilidad a corto plazo de la actual epidemia de influenza AH1N1 en Chile y el mundo.
Universidad de Chile, Santiago

Facultad de Ciencias Departamento de Ciencias Ecológicas.

Departamento de Imaginología. Fundación Arturo López Pérez.

Recibido: 2 de julio de 2009 Aceptado: 22 de enero de 2010

Correspondencia a: Mauricio Canals Lambarri mcanals@uchile.cl. 


\section{Material y Métodos}

Las fuentes de información fueron los reportes diarios de casos totales $\left(Y_{t}\right)$ emitidos por la OMS desde el inicio de la epidemia desde el 25 de abril al 15 de junio de $2009^{7}$ y los reportes actualizados de la situación en Chile emitidos por el Ministerio de Salud entre el 17 de mayo y el 26 de junio de $2009^{10}$.

Hay tres variables básicas útiles para la predicción de casos: el número de casos acumulados $\left(Y_{t}\right)$, el número de casos diarios (incidencia) $(I)$ y la tasa intrínseca de crecimiento del número de casos $\left(R_{t}\right)^{1,9}$. A partir de los casos totales reportados se estimó la incidencia diaria mediante:

$$
I_{t}=Y_{t}-Y_{t-1}
$$

La tasa de crecimiento intrínseca del número de casos corresponde a la tasa de cambio per cápita en el número de casos nuevos en una unidad de tiempo ${ }^{8}$ y se puede estimar mediante:

$$
R_{t}=\left(Y_{t}-Y_{t-1}\right) / Y_{t-1}=I_{t} / Y_{t-1}
$$

A partir de la relación general

$$
\frac{\Delta Y}{\Delta t}=\frac{Y_{t+1}-Y_{t}}{\Delta t}=R_{t} \cdot Y_{t}
$$

haciendo $\Delta t=1$ día, y bajo el supuesto que $R_{t}$ no tendrá grandes variaciones diarias, se puede estimar la incidencia para el día siguiente $\left(I^{*}{ }_{t+1}\right)$ mediante:

$$
I^{*}{ }_{t+1}=R_{t} \cdot Y_{t}
$$

Tomando $\tau=6$ días como una estimación período serial (tiempo medio entre contagio y contagio), basado en otras epidemias del mismo serotipo ${ }^{3}$ se puede considerar que después de 6 días los infectados ya estarán sanos (o muertos) por lo que la población de casos reales y que por tanto son potencialmente contagiantes $\left(Y c_{t}\right)$ se puede estimar mediante:

$$
Y c_{t}=Y_{t}-Y_{t-6}
$$

El número de casos nuevos por cada caso en un período serial es lo que se conoce como tasa o número reproductivo básico de una enfermedad transmisible $\left(R_{0}\right)^{1}$, así bajo el supuesto que $R_{0}$ es constante, se puede estimar que la población $Y c_{t}$ producirá una cantidad $I \tau_{\mathrm{t}}$ en el próximo período serial (6 días):

$$
I \tau_{t}=Y c_{t} \cdot R_{0}
$$

Se pueden obtener dos estimaciones de $R_{0}$, la habitual:

$$
R_{0}=1+R_{t} \tau
$$

o bien con infectados que pueden contagiar muchas personas en un solo instante ("superspreaders"):

$$
R_{0}=1+R_{t} \tau+f(1-f)\left(R_{t} \tau\right)^{2}
$$

donde f corresponde a la razón entre el período infeccioso y el período serial ${ }^{8}$, tomando estos valores como 4,1 y 6 días respectivamente ${ }^{3}$.

Se realizó una regresión al origen entre los valores predichos y observados y se comparó la pendiente con la unidad con una prueba de Student. Así al considerar el observado como variable dependiente y el predicho como variable independiente, una buena predicción se corresponde con una pendiente 1 , una sobreestimación con una pendiente $<1$ y una subestimación con una pendiente $>1$. Además se estudiaron los diagramas de Bland-Altman para comparar predichos y observados ${ }^{11}$ y se calcularon "coeficientes de seguridad" de las predicciones como la razón predicho/observado. Así si la razón predicho/observado es 1,5 se tiene una sobreestimación de $\sim 50 \%$ más de casos.

\section{Resultados}

\section{La epidemia en el mundo}

La tasa intrínseca de crecimiento presentó una inestabilidad en los primeros 10 días, con valores fluctuantes y altos, entre 0,10 y 1,08 , para posteriormente estabilizarse en valores cercanos a 0,1 , lo que sugiere un $\mathrm{R}_{0}$ aproximado de 1,6 (Figura 1). Los valores predichos para el día siguiente presentan un adecuado ajuste con lo observado (Figura 2). Al realizar una regresión entre los valores observados y predichos se obtiene un coeficiente de regresión (pendiente) $\mathrm{B} 1=0,86 \pm 0,06 ; \mathrm{R}^{2}=0,79, \mathrm{~F}_{1,48}$ $=180,6, \mathrm{p}<<0,001$. Sin embargo, la pendiente es menor que $1\left(\mathrm{t}_{48}=2,24, \mathrm{p}<0,05\right)$.

Los casos predichos para los seis días siguientes inicialmente exceden a los realmente observados (Figura 3) para después tener un ajuste mejor. En la regresión entre observados y predichos se obtiene una pendiente $\mathrm{B} 1=$ $0,67 \pm 0,06 ; R^{2}=0,80, F_{1,39}=156,5, p<<0,001$. Sin embargo, la pendiente es menor que $1\left(\mathrm{t}_{39}=6,16, \mathrm{p}<<0,01\right)$.

Los diagramas de Bland-Altman tuvieron una adecuada distribución de los errores para predicciones cercanas al promedio y altas. Sólo se encontraron algunos valores fuera de las bandas de confianza del 95\%, para predicciones bajas. Un diagrama representativo para las predicciones se muestra en la Figura 7.

\section{La epidemia en Chile}

$\mathrm{Al}$ igual que en el mundo, la tasa intrínseca de crecimiento después de valores altos en los primeros días, desciende a valores cercanos a 0,1 , coincidiendo con un valor actual $R_{0}=1,6$ (Figura 4). Los valores predichos para el día siguiente presentan un adecuado ajuste con lo observado (Figura 5). Al realizar una regresión entre los valores observados y predichos se obtiene un coeficiente de regresión (pendiente) $\mathrm{B} 1=0,74 \pm 0,08 ; \mathrm{R}^{2}=0,69$, $\mathrm{F}_{1,37}=81,38, \mathrm{p}<<0,001$. Sin embargo, la pendiente es menor que $1\left(\mathrm{t}_{37}=3,3, \mathrm{p}<0,05\right)$. 


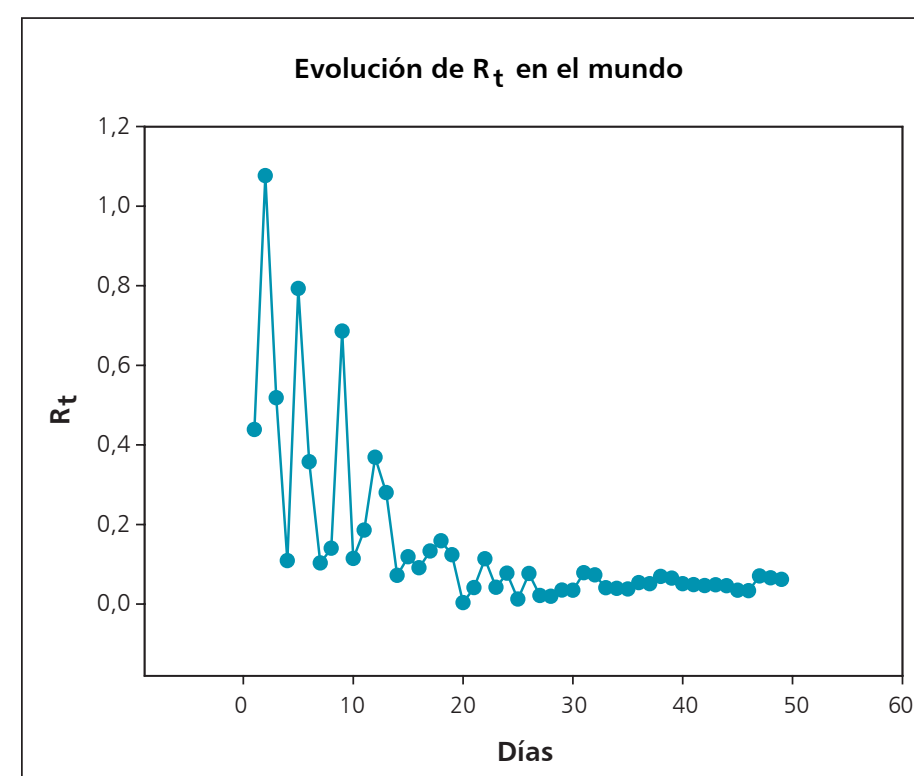

Figura 1. Evolución de la tasa intrínseca diaria de aumento del número de casos $(R)$ de la influenza pandémica AH1N1 en el mundo desde el 25 de abril al 15 de junio de 2009.

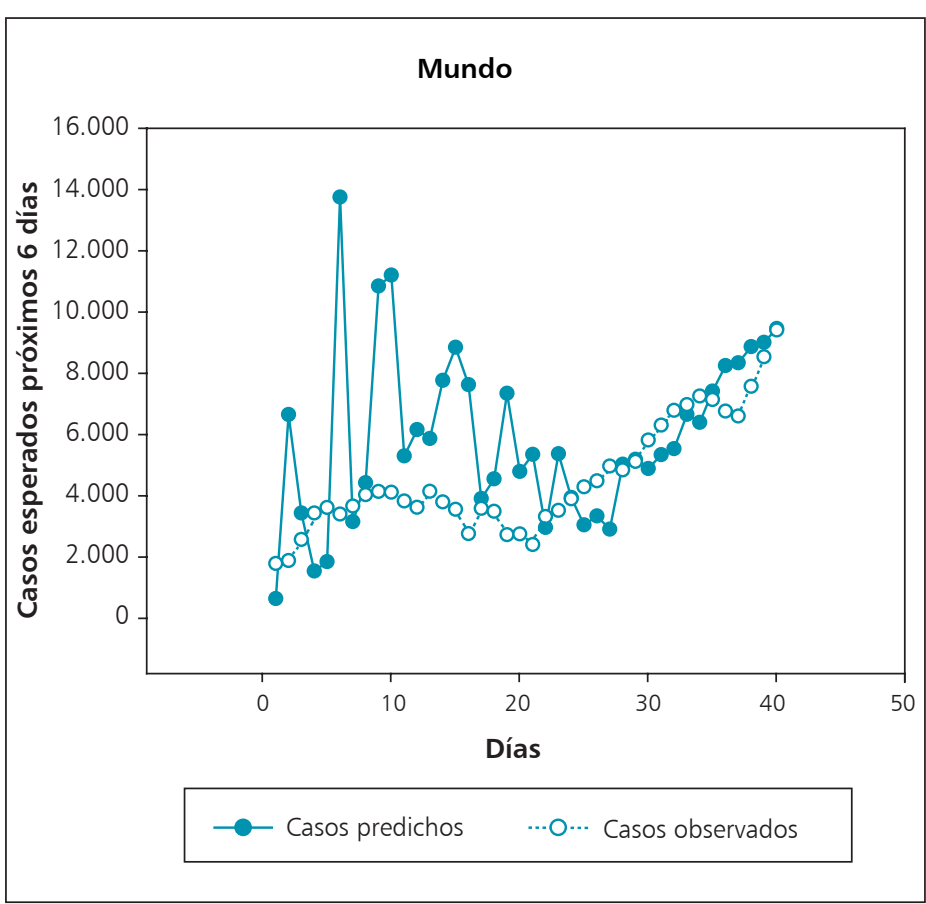

Figura 3. Comparación entre los casos predichos para un período serial y los observados en el mundo en los primeros 50 días de pandemia AH1N1.

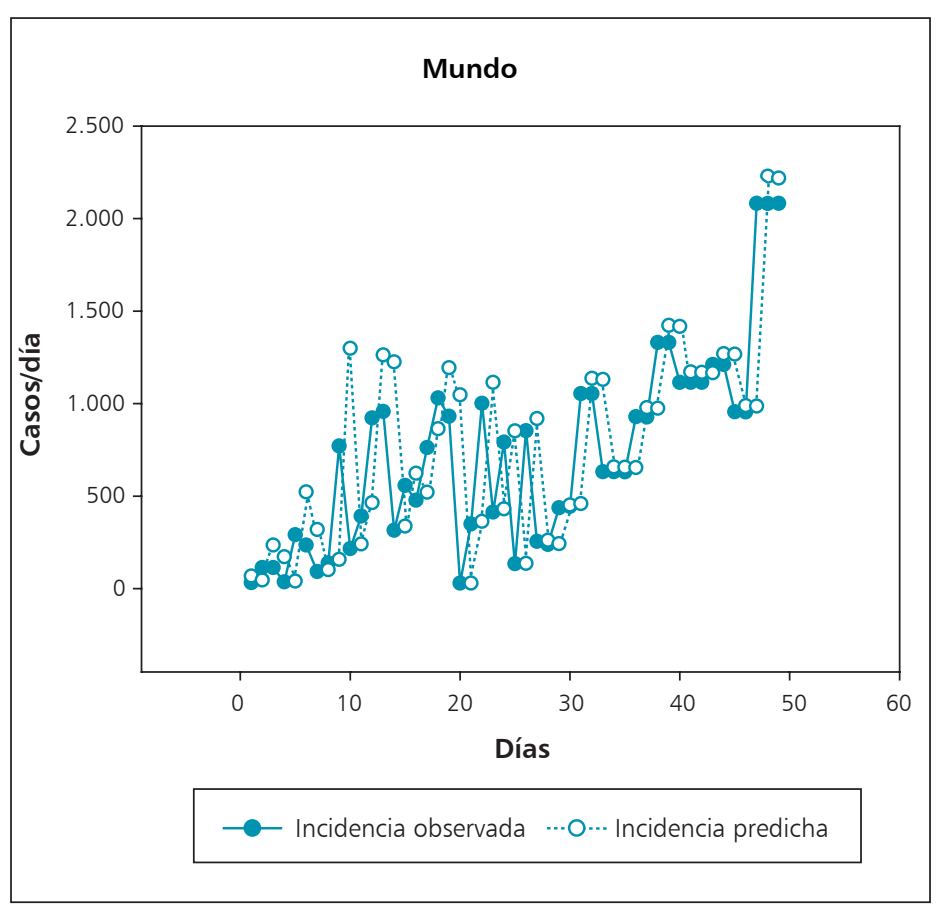

Figura 2. Comparación entre la incidencia mundial (casos/día) de la influenza pandémica AH1N1 observada y predicha en los primeros 50 días de pandemia.

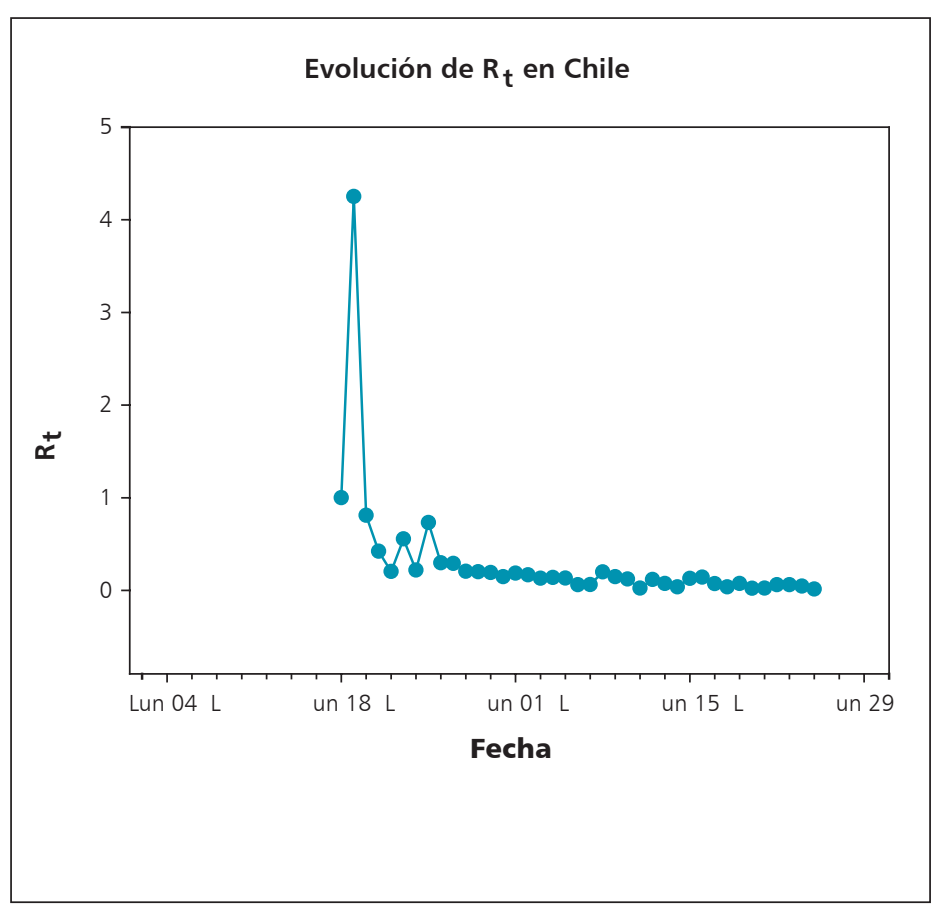

Figura 4. Evolución de la tasa intrínseca diaria de aumento del número de casos de influenza AH1N1 en Chile $\left(R_{t}\right)$ entre el 17 de mayo y el 26 de junio de 2009. 


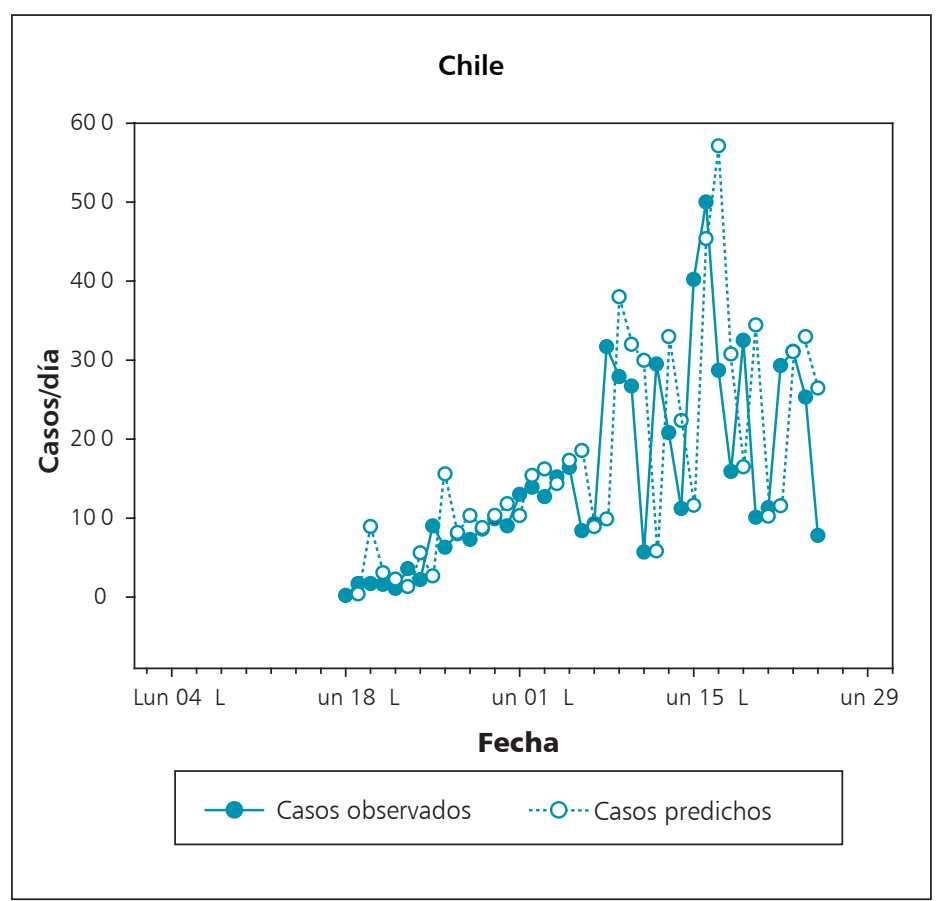

Figura 5. Comparación entre la incidencia (casos/día) observada y predicha de casos de influenza AH1N1 en Chile, hasta en 26 de junio de 2009.

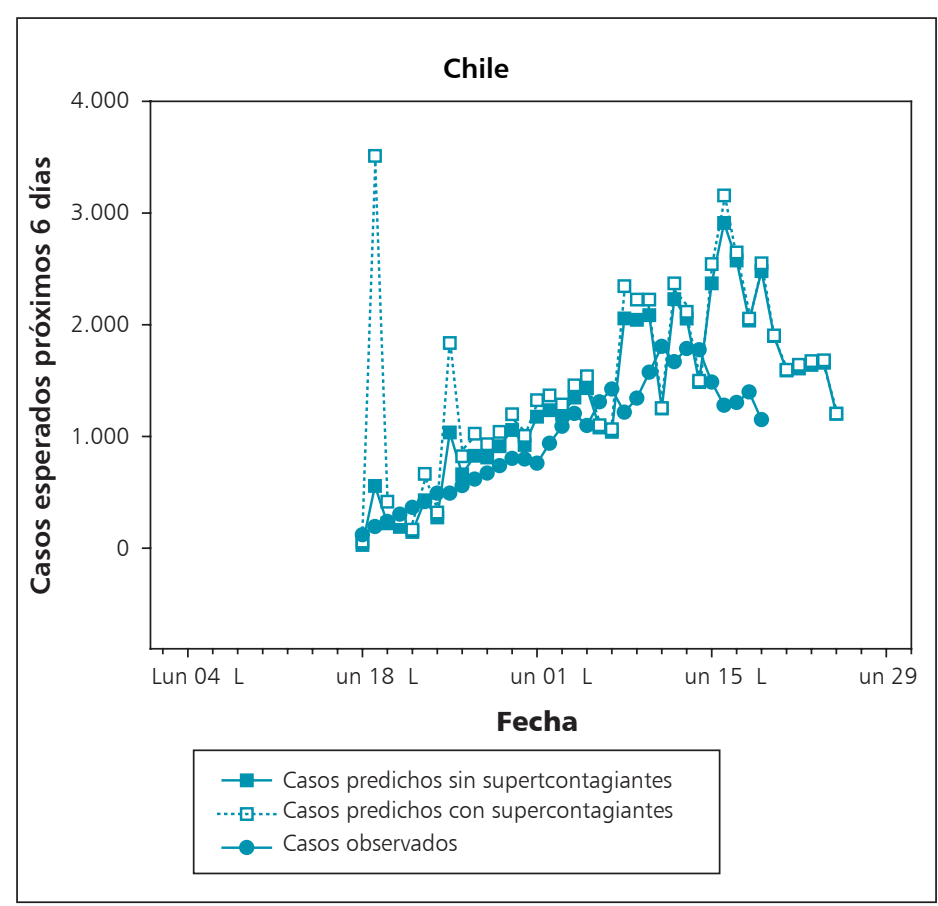

Figura 6. Comparación entre los casos predichos para un período serial y los observados de influenza AH1N1 en Chile hasta el 26 de junio de 2009. Se grafican los casos predichos suponiendo ausencia y presencia de individuos super contagiantes.

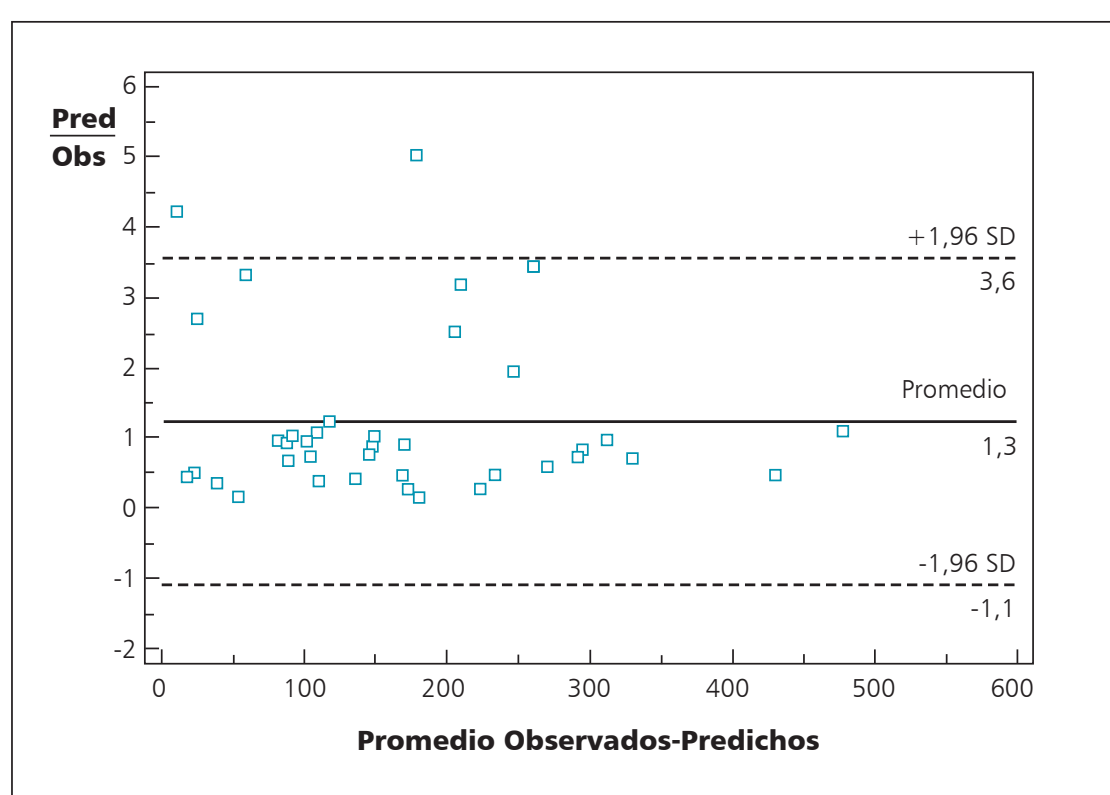

Figura 7. Diagrama de Bland-Altman para la comparación de casos predichos para el próximo período serial y los casos observados de influenza AH1N1 en el mundo. Se grafica la razón entre lo observado y predicho en función del promedio entre ambos. Se aprecia una buena distribución de los errores en las bandas de confianza. Los escasos valores fuera de ellas corresponden a predicciones bajas al inicio de la epidemia lo que coincide con la inestabilidad de la tasa intrínseca de aumento de casos.
Para los casos predichos para los seis días siguiente calculamos las situaciones sin y con super-contagiantes (Figura 6) obteniendo resultados similares:

- Sin supercontagiantes B1 $=0,70 \pm 0,04 ; \mathrm{R}^{2}=0,90$, $\mathrm{F}_{1,32}=278,7, \mathrm{p}<<0,001$. La pendiente es menor que $1\left(\mathrm{t}_{32}=7,5, \mathrm{p}<<0,01\right)$;

- Con supercontagiantes B1 $=0,57 \pm 0,05 ; \mathrm{R}^{2}=0,79$, $\mathrm{F}_{1,32}=119,98, \mathrm{p}<<0,001$. La pendiente es menor que $1\left(\mathrm{t}_{32}=8,6, \mathrm{p}<<0,01\right)$.

Al igual que para el mundo, se encontró una adecuada distribución de los errores para predicciones cercanas al promedio y altas. Sólo se encontraron algunos valores fuera de las bandas de confianza, para predicciones bajas (Figura 7).

\section{Discusión}

Con ciertas variaciones, los modelos epidemiológicos determinísticos básicos trabajan con las fracciones poblacionales de susceptibles (S), infectados (I) y recuperados (R) por lo que son llamados modelos $\mathrm{SIR}^{9}$. En estos modelos la ecuación central es: 
Tabla 1. Tabla base para el cálculo de las estimaciones de todas las variables epidemiológicas de interés en Chile para la epidemia de influenza AH1N1 entre el 17 de mayo y el 26 de junio de 2009. Denomimos " $C_{i, j}$ " al j-ésimo valor de la columna "i"

\begin{tabular}{|c|c|c|c|c|c|c|c|}
\hline Fecha & $C_{1}: Y_{t}$ & $C_{2}: I_{t}$ & $C_{3}: R_{t}$ & $C_{4}: I^{*}{ }_{t+1}$ & $C_{5}: Y c_{t}$ & $C_{6}: I_{t}$ & $C_{7}: I \tau_{t}$ \\
\hline 17-May & 2 & 2 & & & 2 & & \\
\hline 18-May & 4 & 2 & 1,000 & & 4 & 119 & 28,000 \\
\hline 19-May & 21 & 17 & 4,250 & 4,000 & 21 & 192 & 556,500 \\
\hline 20-May & 38 & 17 & 0,810 & 89,250 & 38 & 238 & 222,571 \\
\hline 21-May & 54 & 16 & 0,421 & 30,762 & 54 & 302 & 190,421 \\
\hline 22-May & 65 & 11 & 0,204 & 22,737 & 65 & 364 & 144,444 \\
\hline 23-May & 101 & 36 & 0,554 & 13,241 & 99 & 414 & 427,985 \\
\hline 24-May & 123 & 22 & 0,218 & 55,938 & 119 & 491 & 274,525 \\
\hline 25-May & 213 & 90 & 0,732 & 26,792 & 192 & 491 & $1.034,927$ \\
\hline 26-May & 276 & 63 & 0,296 & 155,854 & 238 & 558 & 660,366 \\
\hline 27-May & 356 & 80 & 0,290 & 81,634 & 302 & 617 & 827,217 \\
\hline 28-May & 429 & 73 & 0,205 & 103,188 & 364 & 671 & 811,843 \\
\hline 29-May & 515 & 86 & 0,200 & 87,969 & 414 & 737 & 911,958 \\
\hline 30-May & 614 & 99 & 0,192 & 103,240 & 491 & 802 & $1.057,318$ \\
\hline 31-May & 704 & 90 & 0,147 & 118,031 & 491 & 796 & 922,824 \\
\hline 01 -Jun & 834 & 130 & 0,185 & 103,192 & 558 & 759 & $1.176,239$ \\
\hline 02-Jun & 973 & 139 & 0,167 & 154,006 & 617 & 937 & $1.234,000$ \\
\hline 03-Jun & 1.100 & 127 & 0,131 & 162,167 & 671 & 1.089 & $1.196,490$ \\
\hline 04-Jun & 1.252 & 152 & 0,138 & 143,577 & 737 & 1.204 & $1.348,040$ \\
\hline 05-Jun & 1.416 & 164 & 0,131 & 173,004 & 802 & 1.097 & $1.432,326$ \\
\hline 06-Jun & 1.500 & 84 & 0,059 & 185,482 & 796 & 1.308 & $1.079,322$ \\
\hline 07-Jun & 1.593 & 93 & 0,062 & 88,983 & 759 & 1.423 & $1.041,348$ \\
\hline 08-Jun & 1.910 & 317 & 0,199 & 98,766 & 937 & 1.218 & $2.055,753$ \\
\hline 09-Jun & 2.189 & 279 & 0,146 & 380,082 & 1.089 & 1.341 & $2.043,443$ \\
\hline 10-Jun & 2.456 & 267 & 0,122 & 319,754 & 1.204 & 1.574 & $2.085,137$ \\
\hline 11-Jun & 2.513 & 57 & 0,023 & 299,567 & 1.097 & 1.804 & $1.249,758$ \\
\hline 12-Jun & 2.808 & 295 & 0,117 & 58,323 & 1.308 & 1.668 & $2.229,273$ \\
\hline 13-Jun & 3.016 & 208 & 0,074 & 329,630 & 1.423 & 1.785 & $2.055,444$ \\
\hline 14-Jun & 3.125 & 112 & 0,037 & 223,407 & 1.215 & 1.774 & $1.485,716$ \\
\hline 15-Jun & 3.527 & 402 & 0,129 & 116,048 & 1.338 & 1.485 & $2.370,722$ \\
\hline 16-Jun & 4.029 & 500 & 0,142 & 453,713 & 1.573 & 1.278 & $2.910,964$ \\
\hline 17-Jun & 4.317 & 287 & 0,071 & 571,165 & 1.804 & 1.354 & $2.575,032$ \\
\hline 18-Jun & 4.476 & 159 & 0,037 & 307,515 & 1.668 & 1.595 & $2.036,606$ \\
\hline 19-Jun & 4.743 & 325 & 0,073 & 164,856 & 1.727 & 1.595 & $2.479,379$ \\
\hline 20-Jun & 4.808 & 101 & 0,021 & 344,387 & 1.683 & 1.869 & $1.898,032$ \\
\hline 21-Jun & 4.921 & 113 & 0,024 & 102,384 & 1.394 & 1.956 & $1.590,575$ \\
\hline 22-Jun & 5.214 & 293 & 0,060 & 115,656 & 1.185 & 1.826 & $1.608,335$ \\
\hline 23-Jun & 5.525 & 363 & 0,070 & 310,445 & 1.208 & 1.663 & $1.712,608$ \\
\hline 24-Jun & 5.778 & 400 & 0,072 & 384,652 & 1.302 & 1.426 & $1.867,575$ \\
\hline 25-Jun & 5.856 & 325 & 0,056 & 418,317 & 1.113 & 1.476 & $1.488,623$ \\
\hline 26-Jun & 6.231 & 375 & 0,064 & 329,387 & 1.423 & 1.289 & $1.969,747$ \\
\hline \\
\hline \multicolumn{8}{|c|}{$\mathrm{C}_{2}$ : número de casos nuevos $\left(I_{t}\right) ; C_{2, j}=C_{1, j}-C_{1, j-1}$} \\
\hline \multicolumn{8}{|c|}{$\mathrm{C}_{3}$ : tasa de crecimiento intrínseca del número de casos $\left(\mathrm{R}_{\mathrm{t}}\right) ; C_{3, j}=C_{2, j} / C_{1, j-1}$} \\
\hline \multicolumn{8}{|c|}{$\mathrm{C}_{4}$ : Casos nuevos predichos para el día siguiente: $\left(I^{*}{ }_{t+1}\right) ; C_{4, j+1}=C_{3, j} \cdot C_{1, j}$} \\
\hline \multicolumn{8}{|c|}{$\mathrm{C}_{5}$ : población de casos potencialmente contagiantes $\left(Y_{c_{1}}\right) ; C_{5, j}=C_{1, j}-C_{1, j-6}$} \\
\hline \multicolumn{8}{|c|}{$\mathrm{C}_{6}$ : casos nuevos acumulados en el próximo período serial $\left(\mathrm{IT}_{\mathrm{t}}\right) ; C_{6, j}=\sum^{j+6} C_{2, j}$} \\
\hline \multicolumn{8}{|c|}{$\mathrm{C}_{7}$ : número de casos estimados a producir en el próximo período serial $(I \tau): C_{7, j}=C_{5, j} \cdot\left(1+C_{3, j}\right)$} \\
\hline
\end{tabular}


$d y / d t=\beta x y-(\gamma+\mu) y \Rightarrow d y / y d t=\beta x-\psi$

donde $x$ e $y$ son los susceptibles e infectados, $\beta$ es la transmisibilidad o coeficiente de transmisión, $\gamma$ la tasa de recuperación, $\mu$ la tasa de mortalidad y $\psi$ la suma de ambas. Si se considera un intervalo de tiempo muy corto (ie "un infinitésimo"), se puede considerar que

$$
d y / y d t=r
$$

lo que es la ecuación de un crecimiento exponencial para este corto intervalo de tiempo. Entonces, la monitorización de la relación $d y / y d t$ conocida como tasa intrínseca de crecimiento permite la predicción a corto plazo para modelos continuos. En este trabajo, consideramos el intervalo de un día, lo que nos permitió analizar la tasa de crecimiento intrínseca discreta del número de casos.

Nuestro enfoque tiene algunas limitaciones. Algunas intrínsecas a la información analizada, que se encuentra basada en los reportes confirmados que no necesariamente representan la situación real de los casos ya que excluyen casos confirmados pero no reportados, casos confirmados en laboratorios no considerados por los sistemas de vigilancia y casos sub-clínicos. Por otra parte, al inicio de las epidemias existe una gran variabilidad estocástica que en nuestro caso queda evidenciada por la inestabilidad inicial en $R t$ (ver Figuras 1 y 4) y por tanto en el cálculo de $R_{0}$ y además la relación entre $\mathrm{Rt}$ y $\mathrm{R}_{0}$ es dependiente del modelo especificado ${ }^{8}$.

A pesar de estas consideraciones, los resultados para Chile y para el mundo son bastante similares. Al inicio de la epidemia existe una inestabilidad de las tasas intrínsecas de crecimiento que pueden ser explicadas por la poca cantidad de casos ya que cambios de muy pocos casos e incluso de un solo caso provocan grandes cambios en el valor de $R_{t}$ y por la inestabilidad por fluctuaciones estocásticas propias del inicio de las epidemias. Por otra parte, los valores iniciales son altos lo que puede ser explicado porque al inicio de la epidemia la tasa de contagios corresponde a la tasa natural sin medidas de contención. Así por ejemplo, en varios países del mundo el $R_{0}$ inicial era cercano a $2^{6}$ mientras cálculos posteriores dan valores entre 1,5 y 1,612. Los valores predichos para el día siguiente y para los seis días siguientes tienen un buen ajuste a lo observado; no obstante, ambos son sobreestimadores. Esto ocurre porque en el caso de la prdicción para el día siguiente se supone que $R_{t}$ es constante, es decir la producción de nuevos casos sigue la dinámica del día anterior (crecimiento exponencial de los casos) y como se puede ver en las Figuras 1 y $3, R_{t}$ al inicio decrece fuertemente. Así, la predicción es mejor cuando se ha estabilizado $R_{t}$. Este argumento es también válido para la predicción a seis días ya que en este caso se supone que
$R_{0}$ permanece constante durante todo el período serial. A pesar que los cálculos de $R_{0}$ se basan en estimaciones del período serial y del período infeccioso obtenidas para la epidemia AH1N1 de 1918-1919, los valores son bastante plausibles; sin embargo, el virus de la actual epidemia es nuevo y el período serial puede ser algo diferente.

Este tipo de modelos tampoco considera variaciones en la transmisibilidad $(\beta)$ que es afectada por cambios estacionales como temperatura y agregación social ${ }^{13-17}$. Sin embargo, al monitorear diariamente las variaciones de $R_{t}$, las incorpora en las predicciones aunque con un retraso de un día o de un período serial según el tipo de predicción.

A pesar de ser relaciones muy simples basadas en la dinámica entre susceptibles infectados y recuperados (modelo SIR), las predicciones son buenas y fáciles de calcular con planillas de cálculo simples como EXCEL (Tabla 1). Como además son sobreestimaciones de los casos reales, tienen un coeficiente de seguridad que permite calcular con cierta holgura las necesidades de salud pública que podrían requerir la atención de los casos que van produciendo. Por ejemplo, en Chile los coeficientes de seguridad (razón predicho/observado) son 1,52 para la predicción al día siguiente, 1,22 para la predicción a 6 días y 2,22 para la predicción a 6 días con super-contagiantes. Para el mundo estos valores son 2,3 1,27 y 1,43 respectivamente.

\section{Resumen}

La influenza AH1N1 se encuentra asolando gravemente a la población de Chile. Tiene transmisibilidad alta, lo que puede afectar la capacidad del sistema de salud por lo que se necesita una adecuada predicción de los posibles casos y complicaciones. Estudiamos los reportes diarios de casos totales emitidos por la OMS y los reportes actualizados de la situación en Chile desde abril a junio de 2009. En este estudio desarrollamos una metodología simple para la predicción a corto plazo de los casos, basada en modelos determinísticos y comparamos los valores predichos con los observados con análisis de regresión y diagramas de Bland-Altman. Encontramos que la tasa intrínseca de crecimiento del número de casos en el mundo y en Chile, después de una inestabilidad y valores iniciales altos, decrece estabilizándose y permitiendo una predicción relativamente buena al día siguiente y a un período serial. Los estimadores que se obtienen son sobre-estimadores, lo que permite un coeficiente de seguridad adecuada para las estimaciones. El método es de implementación rápida en programas computacionales de uso habitual y puede ser de utilidad en el seguimiento de esta epidemia y de otras futuras. 


\section{Referencias}

1.- Canals M, Cattan P E. Zoología médica: Una visión de las especies potencialmente peligrosas desde la perspectiva de la biodiversidad. Generalidades y protozoos. 2006. Editorial Universitaria, Santiago, Chile p 160.

2.- Acha P N, Cifres B. Zoonosis y enfermedades transmisibles comunes al hombre y los animales. II Clamidiosis, rickettsiosis y virosis. OPS publicación científica y técnica 2003; 580 : 329-46.

3.- Mills C E, Robins J M, Lipsitch M. Transmisibility of 1918 pandemic influenza. Nature 2004; 432: 904-6.

4.- Anderson R M, May R M. Population biology of infectious diseases: Part I. Nature 1979; 280 (5721): 361-7.

5.- Wallinga J, Teunis P. Different epidemic curves for severe acute respiratory syndrome reveal similar impacts of control measures. Am J Epidemiol 2004; 160 (6): 509-16.

6.- Canals M. Inicio de la pandemia AH1N1: Algebra, cálculo y geometría del contagio. Rev Med Chile 2009; 137: 852-6.

7.- World Health Organization. 15/6/2009. http:// www.who.int/en/

8.- Heffernan J M, Smith R J, Wahl L M. Perspectives on the basic reproductive ratio. J R Soc Interface 2005; doi: 10.1098/ rsif.2005.0042: 1-13.

9.- Bailey N. The mathematical theory of infectious diseases and its applications. 1975. Griffin, London. p 413.

10.- Ministerio de Salud de Chile. MINSAL. 26/6/2009. http://www.minsal.cl

11.- Altman D G, Bland J M. Measurement in medicine: the analysis of method comparison studies. Statistician 1983; 32: 307-17.

12.- Fraser C, Donnelly C A, Cauchemez S, Hanage W P, Van Kerkhove M D,
Hollingsworth T D, et al. Pandemic potential of a strain of influenza A (H1N1):

Early finding. Science 2009; 324 (5934): 1557-61.

13.- Canals M. Dinámica epidemiológica de la escarlatina en Chile. Rev Chil Pediatr 1989; 60 (1): $15-8$.

14.- Canals M, Martínez L, Firinguetti L. Dinámica ecológica de enfermedades infecciosas. I parte: Estacionalidad. Rev Méd Chile 1989; 117: 87-94.

15.- Canals M, Martínez L, Firinguetti L. Dinámica ecológica de enfermedades infecciosas. II parte: Recurrencia epidémica. Rev Méd Chile 1989; 117: 95-100.

16.- Canals M. Sarampión en Chile: una peligrosa onda. Rev Méd Chile 1992; 120: 585-8.

17.- Canals M. Dinámica de transmisión de enfermedades infecciosas: una expresión particular de una dinámica depredador-presa. Rev Méd Chile 1993; 121: 1316-20.133 